\title{
A Tale of $\beta$-Lactamases and Multidrug Resistance in Pseudomonas aeruginosa Isolated from Inpatients in a University Hospital
}

\author{
Santosh K Yadav ${ }^{1,2}$, Sangita Sharma ${ }^{2}$, Shyam K Mishra², Jeevan B Sherchand ${ }^{2}$
}

\author{
Author(s) affiliation \\ 'Department of Microbiology, \\ Rajarshi Janak University, \\ Janakpurdham, Nepal \\ ${ }^{2}$ Department of Clinical Microbiology, \\ Maharajgunj Medical Campus, \\ Tribhuvan University Teaching \\ Hospital, Institute of Medicine, \\ Kathmandu, Nepal
}

\section{Corresponding author}

Santosh K Yadav, MSc santooshydv@gmail.com

\section{Submitted}

Feb 3, 2021

\section{Accepted}

Mar 29, 2021

\begin{abstract}
\section{Introduction}

In this era of modern medicine, antimicrobial resistance can be regarded as a major health calamity. The emergence of multidrugresistant (MDR) Pseudomonas aeruginosa strains poses therapeutic challenges and lead to treatment failure in hospitalized patients. This study was conducted to determine various types of $\beta$-lactamases among MDR $P$. aeruginosa isolates recovered from hospitalized patients.
\end{abstract}

\section{Methods}

This study was conducted at Tribhuvan University Teaching Hospital, Maharajgunj, Nepal. The clinical samples collected from inpatients were processed for detection of $P$. aeruginosa isolates and antibiotic susceptibility profile was determined. The MDR strains were identified and ceftazidime-resistant isolates were subjected for detection of extended-spectrum- $\beta$-lactamase (ESBL), metallo- $\beta$ lactamase (MBL), and Klebsiella pneumoniae carbapenemase (KPC).

\section{Results}

A total of $161 P$. aeruginosa isolates were recovered during the study period encompassing $73.3 \% \quad(n=118)$ MDR isolates. The MDR isolates included $50.0 \%(n=59)$ from lower respiratory tract infections; and $39.8 \%(n=47)$ were from the intensive care unit patients. The MDR isolates showed a high resistance profile towards piperacillin, cephalosporins, and fluoroquinolones (>85\%). Resistance to carbapenems and aminoglycosides were up to $80 \%$ and $60 \%$ respectively. Extended spectrum- $\beta$-lactamase, MBL, and KPC mediated resistance were seen in $34.7 \%, 43.6 \%$, and $14.4 \%$ MDR isolates, respectively.

\section{Conclusion}

Multidrug resistance as well as resistance mediated by $\beta$-lactamases production were high among $P$. aeruginosa isolates. Therefore, early detection of antimicrobial resistance and rational use of antibiotics play a critical role to fight against this MDR pathogen.

\section{Keywords}

Metallo- $\beta$-lactamase, multidrug resistance, Pseudomonas aeruginosa 


\section{INTRODUCTION}

$P$ eudomonas aeruginosa is one of the emerging hosocomial micro-organisms responsible for a vast variety of infections in hospitalized patients. Various anti-Pseudomonal antibiotics are available for the treatment of these infections but the selection of appropriate antimicrobials is quite challenging because of its ability to gain resistance to multiple classes of antibiotics. ${ }^{1}$ Pseudomonas aeruginosa symbolizes a phenomenon of antimicrobial resistance and practically all known resistance mechanisms are seen in P. aeruginosa isolates. Multidrug-resistant (MDR) phenotypes among $P$. aeruginosa are now common due to the simultaneous expression of different resistance mechanisms. ${ }^{2}$

Carbapenem-resistant $P$ aeruginosa (CRPA) remains an important cause of hospital-acquired infections (HAls) and has been prioritized as a critical pathogen requiring urgent discovery, research, and development of new antibiotics by the World Health Organization (WHO). ${ }^{3}$ Among the various mechanisms of carbapenem resistance in $P$. aeruginosa, the production of carbapenemase enzymes is the most worrying mechanism due to their easily transmissible properties to other bacterial pathogens, increasing prevalence, and their association with resistance to other antimicrobial categories leading to MDR phenotypes. ${ }^{4}$ The isolates producing extended-spectrum- $\beta$-lactamase (ESBL) and metallo- $\beta$-lactamase (MBL) enzymes show resistance to a variety of $\beta$-lactam antibiotics and cause serious infection outbreaks in healthcare settings. ${ }^{5}$

Therefore, this study was designed to detect different types of $\beta$-lactamases among MDR $P$. aeruginosa isolated from hospitalized patients in a tertiary care university hospital.

\section{METHODS}

This study was conducted from January 2017 to December 2017 among the hospitalized patients of Tribhuvan University Teaching Hospital (TUTH), Kathmandu, Nepal. The ethical approval for the study was obtained from the Institutional Review Committee of the Institute of Medicine with reference number 262(6-11-E)2/073/074. In this study, all consecutive and non-replicate isolates of P. aeruginosa recovered from hospitalized patients were included. The various specimens collected from hospitalized patients were cultured on chocolate agar (CA) plate, MacConkey agar (MA) plate, and blood agar (BA) plate and incubated at $37^{\circ} \mathrm{C}$ for 24 to $48 \mathrm{~h}$. Pseudomonas aeruginosa isolates were confirmed by employing different biochemical tests and growing on selective media according to the American Society for Microbiology (ASM). ${ }^{6}$
The antibiotic susceptibility testing of $P$. aeruginosa was performed by the Kirby-Bauer disk diffusion method as recommended by Clinical and Laboratory Standards Institute (CLSI) guidelines (Performance Standards for Antimicrobial Susceptibility Testing, M100S Document, 26th Edition, 2016).7 The antibiotic susceptibility was validated by testing of the reference strain $P$. aeruginosa (ATCC 27853) against all tested antimicrobial agents. The guidelines recommended by Magiorakos et al. ${ }^{8}$ were implemented to identify the MDR and extensively drug-resistant (XDR) isolates.

The MDR $P$. aeruginosa isolates resistant to ceftazidime disk were considered as potential ESBL producers and further tested by the combination disk (CD) method for confirmation of ESBL production. ${ }^{7}$ Modified Hodge test (MHT) was used to detect the production of carbapenemase enzymes in MDR $P$. aeruginosa isolates resistant to either meropenem and/or imipenem. After incubation, a cloverleaf shape indentation growth of Escherichia coli ATCC 25922 within the disk diffusion zone along with the growth of test organism streak was considered as a positive MHT test. A negative MHT test showed inhibition of E. coli ATCC 25922 growth along with the growth of test organism streak. ${ }^{9}$ The MDR $P$. aeruginosa isolates resistant to any carbapenem group of antibiotics were also used to detect and differentiate MBL and KPC enzymes. The detection of MBL-producing, KPC-producing, and $\mathrm{KPC}+\mathrm{MBL}$ co-producing isolates was identified by using a combination of four meropenem disks recommended by Tsakris et al. ${ }^{10}$ The MDR $P$. aeruginosa isolates harboring at least two types of $\beta$-lactamases were characterized as multitype $\beta$-lactamases producing isolates.

The data generated during the study were analyzed by using a 16.0 version of Statistical Package for the Social Sciences (SPSS). Pearson's correlation test was applied to correlate variables where the data with a p-value $\leq 0.05(95 \% \mathrm{Cl})$ was considered statistically significant.

\section{RESULTS}

During the study period, a total of 161 non-duplicate $P$. aeruginosa isolates were recovered from an equal number of consecutive samples. Out of the total $161 P$. aeruginosa, $73.3 \%(n=118)$ were MDR, and about half of the isolates $(50.9 \%)$ were XDR. Out of 118 MDR P. aeruginosa isolates, $66.9 \% \quad(n=79)$ were isolated from male patients and $33.1 \%(n=39)$ were recovered from female patients, with male to female ratio of 2.02. The highest number of MDR isolates $(n=25)$ were from the male patients of the age group 16-32 years (Table 1).

The majority of cases (39.8\%) were from intensive care units followed by the neurology department $(22.0 \%)$ and the minor number was from the 
Table 1. Distribution of MDR Pseudomonas aeruginosa isolates by gender and age group of patients

\begin{tabular}{cccc}
\hline \multirow{2}{*}{$\begin{array}{c}\text { Age group } \\
\text { (Years) }\end{array}$} & \multicolumn{3}{c}{ Number (\%) } \\
\cline { 2 - 4 } & Female & Male & Total \\
\hline$\leq 15$ & $06(5.1)$ & $12(10.2)$ & $18(15.3)$ \\
$16-32$ & $08(6.8)$ & $25(21.2)$ & $33(28.0)$ \\
$33-48$ & $06(5.1)$ & $16(13.5)$ & $22(18.6)$ \\
$49-64$ & $12(10.2)$ & $7(5.9)$ & $19(16.1)$ \\
$\geq 65$ & $7(5.9)$ & $19(16.1)$ & $26(22.0)$ \\
Total & $39(33.1)$ & $79(66.9)$ & $118(100)$ \\
\hline
\end{tabular}

maternity ward $(0.8 \%)$. Similarly, most of the MDR $P$. aeruginosa isolates (50.0\%) were recovered from lower respiratory tract infections followed by pus and swab specimens (20.3\%) while the least number was isolated from each blood and catheter tips samples (1.7\%) (Table 2).

The antibiotic profile showed that the beta-lactams were found to be less effective against MDR $P$. aeruginosa as the highest rate of resistance was mediated towards ceftazidime (96.6\%), cefepime (94.9\%), and piperacillin (96.6\%). More than $85 \%$ of the isolates were resistant to fluoroquinolones (ofloxacin, ciprofloxacin, and levofloxacin), and $72.9 \%$ to gentamicin. About $80 \%$ of the isolates were resistant to carbapenems, whereas amikacin showed promising efficacy $(49.2 \%$ isolates were susceptible). Polymyxin B was found to be the most effective regimen against MDR $P$. aeruginosa as resistance was not documented towards this antibiotic (Table 3).

The antibiotic resistance mediated by $\beta$-lactamase enzymes was frequently seen in this study. Resistance due to ESBL and MBL production
Table 3. Antibiotic susceptibility profile of MDR Pseudomonas aeruginosa isolates

\begin{tabular}{lcc}
\hline \multirow{2}{*}{\multicolumn{1}{c}{ Antibiotics }} & \multicolumn{2}{c}{ Number (\%) } \\
\cline { 2 - 3 } & Sensitive & Resistant \\
\hline Piperacillin & $4(3.4)$ & $114(96.6)$ \\
Piperacillin-tazobactam & $25(21.2)$ & $93(78.8)$ \\
Ceftazidime & $4(3.4)$ & $114(96.6)$ \\
Cefepime & $6(5.1)$ & $112(94.9)$ \\
Ciprofloxacin & $3(2.5)$ & $115(97.5)$ \\
Ofloxacin & $5(4.2)$ & $113(95.8)$ \\
Levofloxacin & $14(11.9)$ & $104(88.1)$ \\
Gentamicin & $32(27.1)$ & $86(72.9)$ \\
Amikacin & $58(49.2)$ & $60(50.8)$ \\
Meropenem & $19(16.1)$ & $99(83.9)$ \\
Imipenem & $25(21.2)$ & $93(78.8)$ \\
Polymyxin B & $118(100)$ & $0(0)$ \\
\hline
\end{tabular}

was observed in $34.7 \%$ and $63.6 \%$ MDR isolates, respectively. The highest rate of both ESBL and MBL mediated resistance was seen in MDR isolates from catheter tips (100\% each). The KPC mediated antibiotic resistance was reported in $14.4 \%$ of MDR isolates, with the highest rate of isolates being from body fluid samples (21.4\%). MBL with ESBL and $\mathrm{MBL}$ with KPC type $\beta$-lactamases production were documented in $14.4 \%$ and $7.6 \%$ isolates, respectively (Table 4).

Among carbapenem-resistant MDR isolates $(n=99)$, MHT was seen positive in $67.7 \%(n=67)$ and negative in $32.3 \%(n=32)$ isolates. Among the MBL producing MDR isolates, $78.7 \%$ were $\mathrm{MHT}$ positive. Additionally, 33.3\% of MBL non-producing MDR isolates were MHT positive. Similarly, all KPC producers and MBL+KPC co-producing MDR isolates were MHT positive (Table 5).

Table 2. Ward and specimen wise distribution of MDR Pseudomonas aeruginosa isolates

\begin{tabular}{lccccccc}
\hline \multirow{2}{*}{ Wards } & \multicolumn{7}{c}{ Number of isolates in different specimens } \\
\cline { 2 - 8 } & LRTS & Pus/swabs & Urine & Body fluids & Blood & Catheter tips & Total (\%) \\
\hline ICU $^{\text {b }}$ & 32 & 5 & 1 & 7 & 1 & 1 & $47(39.8)$ \\
Neurology & 17 & 2 & 7 & 0 & 0 & 0 & $26(22.0)$ \\
Surgery & 4 & 8 & 6 & 5 & 0 & 1 & $24(20.3)$ \\
Respiratory & 4 & 0 & 1 & 0 & 0 & 0 & $5(4.2)$ \\
Pediatrics & 0 & 2 & 1 & 1 & 0 & 0 & $4(3.4)$ \\
Orthopedics & 1 & 1 & 1 & 0 & 0 & 0 & $3(2.6)$ \\
Burn & 0 & 3 & 0 & 0 & 0 & 0 & $3(2.6)$ \\
ENT & 0 & 3 & 0 & 0 & 0 & 0 & $3(2.6)$ \\
Nephrology & 1 & 0 & 0 & 1 & 0 & 0 & $2(1.7)$ \\
Maternity & 0 & 0 & 0 & 0 & 1 & 0 & $1(0.8)$ \\
$\quad$ Total (\%) & $59(50.0)$ & $24(20.3)$ & $17(14.4)$ & $14(11.9)$ & $2(1.7)$ & $2(1.7)$ & $118(100)$ \\
\hline
\end{tabular}

aLower respiratory tract specimens (sputum, bronchoalveolar lavage, and endotracheal aspirate); ${ }^{b}$ ntensive care units; ' ${ }^{\mathrm{E} a r}$, nose, and throat 
Table 4. Distribution of $\beta$-lactamases producing MDR

Pseudomonas aeruginosa isolates in different specimens

\begin{tabular}{|c|c|c|c|c|c|c|}
\hline \multirow{2}{*}{$\begin{array}{l}\text { Specimen } \\
\text { type }\end{array}$} & \multirow{2}{*}{ Number } & \multicolumn{5}{|c|}{ Number and types of $\beta$-lactamases producing isolates (\%) } \\
\hline & & $\mathrm{ESBL}^{\mathrm{b}}$ & $M B L^{c}$ & $\mathrm{KPC}^{\mathrm{d}}$ & $\mathrm{MBL}+\mathrm{ESBL}$ & $M B L+K P C$ \\
\hline LRTS $^{a}$ & 59 & $22(37.3)$ & $37(62.7)$ & 9 (15.3) & $10(16.9)$ & $5(8.5)$ \\
\hline Pus/swabs & 24 & $5(20.8)$ & $17(70.8)$ & $2(8.3)$ & $2(8.3)$ & $0(0)$ \\
\hline Urine & 17 & $5(29.4)$ & $9(52.9)$ & $3(17.6)$ & $1(5.9)$ & $2(11.8)$ \\
\hline Body fluids & 14 & $6(42.9)$ & $9(64.3)$ & $3(21.4)$ & $2(14.3)$ & $2(14.3)$ \\
\hline Blood & 2 & $1(50.0)$ & $1(50.0)$ & $0(0)$ & $0(0)$ & $0(0)$ \\
\hline Catheter tip & 2 & $2(100)$ & $2(100)$ & $0(0)$ & $2(0)$ & $0(0)$ \\
\hline Total & 118 & 41 (34.7) & 75 (63.6) & $17(14.4)$ & $17(14.4)$ & $9(7.6)$ \\
\hline
\end{tabular}

a Lower respiratory tract specimen (sputum, bronchoalveolar lavage, and endotracheal aspirate);

${ }^{\mathrm{b}}$ Extended-spectrum- $\beta$-lactamase; ${ }^{c}$ Metallo- $\beta$-lactamase; ${ }^{d K l e b s i e l l a ~ p n e u m o n i a e ~ c a r b a p e n e m a s e ~}$

Table 5. Correlation between Modified Hodge Test and carbapenemase enzymes in carbapenem-resistant $(n=99)$ MDR isolates

\begin{tabular}{lcccc}
\hline \multirow{2}{*}{$\begin{array}{c}\text { Carbapenemases } \\
\text { production }\end{array}$} & Number & \multicolumn{2}{c}{ Modified Hodge Test } & \multirow{2}{*}{ p-value } \\
\cline { 3 - 4 } & & Positive (\%) & Negative (\%) & \\
\hline MBL & & & & \\
Producer & 75 & $59(78.7)$ & $16(21.3)$ & $<0.001$ \\
Non-producer & 24 & $8(33.3)$ & $16(66.67)$ & \\
KPC & & & & 0.002 \\
Producer & 17 & $17(100)$ & $0(0)$ & \\
Non-producer & 82 & $50(61.0)$ & $32(39.0)$ & \\
MBL+KPC & & & & 0.03 \\
Co-producer & 9 & $9(100)$ & $0(0)$ & \\
Non-coproducer & 90 & $58(64.4)$ & $32(35.6)$ & \\
\hline
\end{tabular}

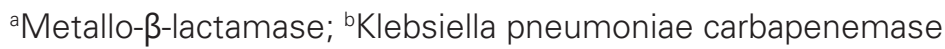

\section{DISCUSSION}

In Nepalese hospitals, antibiotic resistance among micro-organisms is a major problem. ${ }^{11}$ In the present study, we have isolated a highest number of MDR $P$. aeruginosa isolates from lower respiratory tract infections (50.0\%). The highest recovery rate of $P$. aeruginosa from lower respiratory tract infections in our study also correlates with other studies done in various countries. ${ }^{12,13}$ We documented a higher number of MDR $P$. aeruginosa (39.8\%) isolates from ICU patients. In another study, $P$. aeruginosa was also documented to be the usual pathogen isolated from ICU patients than that of other hospital wards. ${ }^{14}$

An increasing trend of resistance to multiple classes of antibiotics has been documented in Pseudomonas aeruginosa, which is the crucial reason for treatment failure in hospitalized patients. ${ }^{15}$ Multidrugresistant $P$. aeruginosa isolates were found highly resistant to several classes of antibiotics. From previous literature, an elevated rate of resistance against carbapenems, fluoroquinolones, and third- generation cephalosporins has been documented in this bacterium. ${ }^{13}$ As a consequence of the arbitrary use of antibiotics, these agents have decreased their usefulness in Nepal. ${ }^{11}$ Among the $\beta$-lactam group, $96.6 \%$ MDR isolates were found to be resistant to ceftazidime, $94.9 \%$ to cefepime, $96.6 \%$ to piperacillin, $78.8 \%$ to piperacillin-tazobactam, $83.9 \%$ to meropenem, and $78.8 \%$ to imipenem. Mishra et al. ${ }^{16}$ from Nepal have documented a lower rate of resistance towards beta-lactams. The findings of our study correlate with the observations of Parajuli et al., ${ }^{11}$ and Ansari et al. ${ }^{15}$ from Nepal, and Behera et al. ${ }^{17}$ from India. We identified a resistance rate of $97.5 \%$ to ciprofloxacin, $95.8 \%$ to ofloxacin, and $88.1 \%$ to levofloxacin, which is higher than previous reports from Nepal by Mishra et al. ${ }^{16}$ in 2008 and Anil et al. ${ }^{18}$ in 2013. Parajuli et al. ${ }^{11}$ from Nepal reported a similar rate of fluoroquinolone resistance in $P$. aeruginosa (up to 90\%). The increasing trend of resistance was also reported against aminoglycosides, being resistance rate up to $72.9 \%$ to gentamicin and $50.8 \%$ to amikacin and is higher than that reported by Mishra et al. ${ }^{16}$ in 2008 
but similar to the report of Parajuli et al. ${ }^{11}$ in 2017 in ICU patients. In this study, polymyxin B was found to be $100 \%$ effective against MDR $P$. aeruginosa, which could be used as a therapeutic option against MDR P. aeruginosa isolates. However, it could arise detrimental consequences in hospitalized patients due to its toxicity. Other studies also found polymyxin $B$ as the most effective regimen against serious pseudomonal infections. ${ }^{11,15}$

The rate of MDR in $P$. aeruginosa isolates has dramatically increased (73.3\% MDR isolates) and $50.9 \%$ isolates were $\mathrm{XDR}$, which is higher than those reported by Mishra et al. ${ }^{16}$ (65.9\% MDR) from Nepal in 2008, Prakash et al. ${ }^{19}$ (31.7\% MDR) from India in 2014, Flamm et al. ${ }^{20}$ (24.9\% MDR) and Langeveld et al. ${ }^{21}$ (37.3\% MDR) from the USA in 2004 and 2017 respectively, and Pereira et al. ${ }^{22}$ (39.9\% MDR and 2.9\% XDR) from Portugal in 2015. The rate of MDR P. aeruginosa is in an upward trend throughout the world, especially in developing countries causing a life-threatening situation.

In this study, we observed $34.7 \%$ ESBL producing MDR isolates. Umadevi et al. ${ }^{23}$ from India reported only $13 \%$ ESBL producing $P$. aeruginosa isolates. Likewise, in two other studies from India ${ }^{24}$ and Brazil, ${ }^{25}$ low rates of ESBL production of about $21 \%$ were observed. The variation in the prevalence of ESBL from country to country and hospital to the hospital may be ascribed to antibiotic prescribing habits and the presence of pathogens harboring ESBL genes.

World Health Organization published a list of antibiotic-resistant bacteria and categorized carbapenem-resistant P. aeruginosa (CRPA) as a critical pathogen (priority first). ${ }^{3}$ The emergence of MBLs in $P$. aeruginosa is becoming a serious summons as these enzymes possess high hydrolytic activity against all $\beta$-lactam classes. ${ }^{23,26}$ Among a total of $118 \mathrm{MDR}$ isolates, we identified $63.6 \%$ isolates as MBL producers which is much higher than previous studies carried out by different authors from Nepal. ${ }^{15,16}$ However, a lower rate of MBL production was reported by Joseph et al. ${ }^{27}$ (20\%) from India, Rafiee et al. ${ }^{28}$ (37.3\%) from Iran, and only 3.3\% by Mishra et al. ${ }^{16}$ from Nepal in 2008. We have found $14.4 \%$ KPC-producing isolates and they were also co-producing MBL enzymes. Falahat et al. ${ }^{29}$ reported $12 \%$ KPC-producing $P$. aeruginosa from Iran. We have also identified a few MDR isolates harboring a multitype of $\beta$-lactamase enzymes. MBL with ESBL enzymes was identified in $14.4 \%$ and $\mathrm{MBL}$ with $\mathrm{KPC}$ was seen in $7.6 \%$ MDR isolates. Besides, $14.36 \%$ co-producer of ESBL and MBL was documented in P. aeruginosa by Chaudhary and Payasi ${ }^{30}$ from India while only $3.4 \%$ co-producer of ESBL and MBL was reported by Ansari et al..$^{15}$ from Nepal. In the present study, we found infections in hospitalized patients with highly resistant $P$. aeruginosa isolates. This is due to the high prevalence of MDR strains and the production of various beta-lactamases that can contribute resistance to different classes of antibiotics.

\section{CONCLUSION}

According to the findings of this study, infections caused by MDR $P$. aeruginosa in hospitalized patients are common. MDR isolates had a higher rate of $E S B L$ and $M B L$ production and these isolates were only completely susceptible to potentially toxic antibiotics like polymyxin $B$, which is a major concern for hospitalized patients. Therefore, early detection of antimicrobial resistance and rational use of antibiotics play a critical role to fight against this MDR pathogen.

\section{ACKNOWLEDGEMENTS}

The authors would like to appreciate all staff of the Clinical Microbiology Department of Tribhuvan University Teaching Hospital for their generous support during the study.

\section{CONFLICT OF INTEREST}

None declared.

\section{REFERENCES}

1. Bajpai V, Govindaswamy A, Khurana $S$, et al. Phenotypic and genotypic profile of antimicrobial resistance in Pseudomonas species in hospitalized patients. Indian J Med Res. 2019 Feb 1;149(2):216-21.

2. Lister $P$, Wolter $D$, Hanson N. Antibacterial-Resistant Pseudomonas aeruginosa: Clinical Impact and Complex Regulation of Chromosomally Encoded Resistance Mechanisms. Clin Microbiol Rev. 2009;22(4):582-610.

3. Tacconelli E, Carrara E, Savoldi A, et al. Discovery, research, and development of new antibiotics: the WHO priority list of antibioticresistant bacteria and tuberculosis. Lancet Infect Dis. $2018 \mathrm{Mar}$ 1;18(3):318-27

4. Gniadek TJ, Carroll KC, Simner PJ. Carbapenem-Resistant NonGlucose-Fermenting Gram-Negative Bacilli: the Missing Piece to the Puzzle. J Clin Microbiol. 2016;54(7):1700-10.

5. Livermore D. Multiple Mechanisms of Antimicrobial Resistance in Pseudomonas aeruginosa: Our Worst Nightmare? Clin Infect Dis. 2002;34(5):634-40.

6. Isenberg H. Clinical Microbiology Procedure Handbook. Second Edi. American Society for Microbiology (ASM), Washington, DC, USA; 2007.

7. CLSI. Performance Standards for Antimicrobial Susceptibility Testing; Twenty-Sixth Informational Supplement. CLSI Document M100S. 26th ed. Clinical and Laboratory Standards Institute, Wayne, PA, USA; 2016

8. Magiorakos A, Srinivasan A, Carey R, et al. Multidrug-resistant, extensively drug-resistant and pandrug-resistant bacteria: an international expert proposal for interim standard definitions for acquired resistance. Clin Microbiol Infect. 2012;18(3):268-81.

9. Amjad A, Mirza I, Abbasi S, et al. Modified Hodge test: A simple and effective test for detection of carbapenemase production. Iran J Microbiol. 2011;3(4):189-93.

10. Tsakris A, Poulou A, Pournaras $S$, et al. A simple phenotypic 
method for the differentiation of metallo- $\beta$-lactamases and class A KPC carbapenemases in Enterobacteriaceae clinical isolates. J Antimicrob Chemother. 2010;65(8):1664-71.

11. Parajuli NP, Acharya SP, Mishra SK, et al. High burden of antimicrobial resistance among gram negative bacteria causing healthcare associated infections in a critical care unit of Nepal. Antimicrob Resist Infect Control. 2017 Jun;6(1):67.

12. Rit K, Nag F, Raj H, et al. Prevalence and Susceptibility Profiles of Nonfermentative Gram-negative Bacilli Infection in a Tertiary Care Hospital of Eastern India. Indian J Clin Pract. 2013;24(5):451-5.

13. Fatima $A$, Naqvi $S$, Khaliq $S$, et al. Antimicrobial susceptibility pattern of clinical isolates of Pseudomonas aeruginosa isolated from patients of lower respiratory tract infections. Springerplus. 2012;1(1):70.

14. Datta P, Rani H, Chauhan $R$, et al. Health-care-associated infections: Risk factors and epidemiology from an intensive care unit in Northern India. Indian J Anaesth. 2014;58(1):30-5.

15. Ansari S, Dhital R, Shrestha $S$, et al. Growing Menace of Antibacterial Resistance in Clinical Isolates of Pseudomonas aeruginosa in Nepal: An Insight of Beta-Lactamase Production. Biomed Res Int. 2016;2016.

16. Mishra S, Acharya J, Kattel $H$, et al. Metallo-beta-lactamase producing gram-negative bacterial isolates. J Nepal Health Res Counc. 2012;10(22):208-13.

17. Behera B, Mathur P, Das A, et al. An evaluation of four different phenotypic techniques for detection of metallo- $\beta$-lactamase producing Pseudomonas aeruginosa. Indian J Med Microbiol. 2008;26(3):233-7.

18. Anil C, Shahid R. Antimicrobial Susceptibility Patterns of Pseudomonas aeruginosa Clinical Isolates at a Tertiary Care Hospital in Kathmandu, Nepal. Asian J Pharm Clin Res. 2013;6(3):235-8.

19. Prakash $V$, Mishra P, Premi $H$, et al. Increasing incidence of multidrug resistant Pseudomonas aeruginosa in inpatients of a tertiary care hospital. Int J Res Med Sci. 2014;2(4):1302-06.

20. Flamm R, Weaver M, Thornsberry C, et al. Factors associated with relative rates of antibiotic resistance in Pseudomonas aeruginosa isolates tested in clinical laboratories in the United States from 1999 to 2002. Antimicrob Agents Chemother. 2004;48(7):2431-6.
21. Langeveld I, Gagnon R, Conrad P, et al. Multiple-Drug Resistance in Burn Patients: A Retrospective Study on the Impact of Antibiotic Resistance on Survival and Length of Stay. J Burn Care Res. 2017;38(2):99-105.

22. Pereira S, Marques M, Pereira J, et al. Multidrug and Extensive Drug Resistance in Pseudomonas aeruginosa Clinical Isolates from a Portuguese Central Hospital: 10-Year Survey. Microb Drug Resist. 2015;21(2):194-200.

23. Umadevi $S$, Joseph N, Kumari $K$, et al. Detection of Extended Spectrum Beta Lactamases, Ampc Beta Lactamases and Metallo betalactamases in Clinical Isolates of Ceftazidime Resistant Pseudomonas aeruginosa. Brazilian J Microbiol. 2011;42(4):12848.

24. Aggarwal R, Chaudhary U, Bala K. Detection of extended-spectrum $\beta$-lactamase in Pseudomonas aeruginosa. Indian J Pathol Microbiol. 2008;51(2):222-4.

25. Picao R, Poirel L, Gales A, et al. Diversity of beta-lactamases produced by ceftazidime-resistant Pseudomonas aeruginosa isolates causing bloodstream infections in Brazil. Antimicrob Agents Chemother. 2009;53(9):3908-13.

26. Cornaglia G, Giamarellou H, Rossolini GM, et al. Metallo- $\beta$ lactamases: a last frontier for $\beta$-lactams? Lancet Infect Dis. 2011;11(5):381-93.

27. Joseph N, Sistla S, Dutta T, et al. Ventilator-associated pneumonia in a tertiary care hospital in India: Role of multi-drug resistant pathogens. J Infect Dev Ctries. 2010;4(4):218-25.

28. Rafiee $R$, Eftekhar $F$, Tabatabaei $S$, et al. Prevalence of ExtendedSpectrum and Metallo $\beta$-Lactamase Production in AmpC $\beta$-Lactamase Producing Pseudomonas aeruginosa Isolates From Burns. Jundishapur J Microbiol. 2014;7(9):e16436.

29. Falahat S, Shojapour M, Sadeghi A. Detection of KPC carbapenemase in Pseudomonas aeruginosa Isolated from clinical samples using modified hodge test and boronic acid phenotypic methods and their comparison with the polymerase chain reaction. Jundishapur J Microbiol. 2016;9(9):e27249.

30. Chaudhary M, Payasi A. Rising Antimicrobial Resistance of Pseudomonas aeruginosa Isolated from Clinical Specimens in India. J Proteomics Bioinforma. 2013;6(1):5-9. 